\title{
Preoperative Concurrent Chemoradiotherapy for Locally Advanced Rectal Cancer: Treatment Outcomes and Analysis of Prognostic Factors
}

\author{
Moonkyoo Kong, $\mathrm{MD}^{1}$ \\ Seong Eon Hong, MD \\ Woo Suk Choi, MD2 \\ Si-Young Kim, MD³ \\ Jinhyun Choi, MD'
}

Departments of ${ }^{1}$ Radiation Oncology, ${ }^{2}$ Diagnostic Radiology, and ${ }^{3}$ Internal Medicine, Kyung Hee University School of Medicine, Seoul, Korea

\begin{abstract}
Purpose
This study was designed to investigate the long-term oncologic outcomes for locally advanced rectal cancer patients after treatment with preoperative concurrent chemoradiotherapy followed by total mesorectal excision, and to identify prognostic factors that affect survival and pathologic response.
\end{abstract}

\section{Materials and Methods}

From June 1996 to June 2009, 135 patients with locally advanced rectal cancer were treated with preoperative concurrent chemoradiotherapy followed by total mesorectal excision at Kyung Hee University Hospital. Patient data was retrospectively collected and analyzed in order to determine the treatment outcomes and identify prognostic factors for survival.

\section{Results}

The median follow-up time was 50 months (range, 4.5 to 157.8 months). After preoperative chemoradiotherapy, sphincter preservation surgery was accomplished in $67.4 \%$ of whole patients. A complete pathologic response was achieved in $16 \%$ of patients. The estimated 5- and 8-year overall survival, loco-regional recurrence-free survival, and distant metastasis-free survival rate for all patients was $82.7 \%$ and $75.7 \%, 76.8 \%$ and $71.9 \%, 67.9 \%$ and $63.3 \%$, respectively. The estimated 5- and 8-year overall survival, loco-regional recurrence-free survival, and distant metastasis-free survival rate for pathologic complete responders was $100 \%$ and $100 \%, 100 \%$ and $88.9 \%$, $95.5 \%$ and $95.5 \%$, respectively. In the multivariate analysis, pathologic complete response was significantly associated with overall survival. The predictive factor for pathologic complete response was pretreatment clinical stage.

\section{Conclusion}

Preoperative chemoradiotherapy for locally advanced rectal cancer resulted in a high rate of overall survival, sphincter preservation, down-staging, and pathologic complete response. The patients achieving pathologic complete response had very favorable outcomes. Pathologic complete response was a significant prognostic factor for overall survival and the significant predictive factor for a pathologic complete response was pretreatment clinical stage.

\section{Key words}

Rectal neoplasms, Preoperative care, Chemoradiotherapy, Pathologic complete response

\section{Introduction}

Preoperative chemoradiotherapy (CRT) followed by radical surgery is widely accepted as the standard treatment for locally advanced rectal cancer. The theoretical advantages of preoperative CRT include improved compliance with the CRT regimen, if it is administered prior to radical surgery, a well-defined tumor target with an intact tumor oxygen supply that can maximize tumor response, the absence of postoperative surgical changes that can minimize radiation-induced normal tissue toxicity, and
CRT-induced tumor down-staging which may enhance sphincter preservation in patients with low-lying tumors [1,2]. In addition, preoperative CRT provides an early endpoint for treatment results with prognostic value [3]. However, preoperative CRT also has disadvantages including a possible increase in postoperative complications [1].

The large number of studies regarding preoperative CRT for locally advanced rectal cancer that have been published in the last several years have demonstrated the efficacy of this treatment for complete pathologic response, tumor down-staging, and enhanced sphincter preservation [4,5]. Encouraging results have also been achieved in terms of recurrence rate, 
and survival $[2,6,7]$. However, the reported rates of sphincter preservation after preoperative CRT vary widely, and the impact of treatment response on survival is highly controversial. A number of previous studies $[1,2,5,8,9]$ have attempted to identify clinical and pathological factors associated with survival and the risk of recurrence after preoperative CRT. These studies produced contradicting results for the identification of independent prognostic factors.

In order to contribute to the resolution of these contradictions, we report the long-term oncologic outcomes for patients with locally advanced rectal cancer after preoperative concurrent CRT followed by total mesorectal excision (TME) at our institution, and identify prognostic factors affecting survival and pathologic response.

\section{Materials and Methods}

\section{Patient population}

The patients eligibility criteria included: 1) histologically confirmed rectal adenocarcinoma, 2) clinically diagnosed T3-4 or node-positive disease, 3) no distant metastasis, 4) no prior chemotherapy, 5) no prior radiotherapy (RT) in pelvic cavity, 6) preoperative concurrent CRT, 7) TME of curative aim after concurrent CRT, 8) follow-up period greater than 6 months after curative TME, 9) no other simultaneous malignancies. At Kyung Hee University Hospital, 135 rectal cancer patients met the eligibility criteria between June 1996 and June 2009, and were enrolled in this study.

\section{Clinical evaluation}

Data collected retrospectively for each patient included: age, gender, tumor distance from anal verge, pretreatment tumor size, histologic grade, tumor circumference extent, pretreatment clinical tumor, node and metastasis (TNM) stage, RT dose, chemotherapy regimen, type of surgery (sphincter preservation surgery or abdominoperineal resection), interval of time between CRT and surgery, pathologic TNM stage, date and site of tumor recurrence, date and status at last follow-up visit. The tumor distance from anal verge was defined as the distance from the caudal tumor edge to the anal verge, and was assessed by digital examination and sigmoidoscopy. Pretreatment tumor size was defined as the longest diameter in any dimension, and pretreatment clinical staging was performed using a combination of physical examination, computed tomography, magnetic resonance imaging and endorectal ultrasonography. The clinical and pathologic TNM stages were determined according to the American Joint Committee on Cancer TNM staging system (7th edition), and the histologic grade of adenocarcinoma was described according to the World Health Organization classification.

\section{Treatments}

All patients received 5-fluorouracil (5-FU)-based CRT. Forty one patients received continuous 5-FU infusion and 94 patients received bolus 5-FU with leucovorin. RT began on the first day of chemotherapy and was administered 5 times per week with a daily fraction of $180 \mathrm{cGy}$. Initially, the entire pelvis was treated with 3- or 4-field techniques to 4,500 cGy in a supine position. The superior border of the entire pelvis was placed at the lumbosacral junction. The inferior border was placed at $>$ $2 \mathrm{~cm}$ caudal to the gross tumor. The lateral field border was $1.5 \mathrm{~cm}$ outside the bony pelvic inlet. The anterior border of the lateral fields was $\sim 3 \mathrm{~cm}$ anterior to the gross tumor and shaped to include the internal iliac lymph nodes if T3, and external iliac lymph nodes if T4. The posterior border of the lateral fields was extended to encompass the bony sacrum. Most patients received a boost to the primary tumor bed for a median total dose of 5,040 cGy. A two-dimensional conventional technique or three-dimensional conformal technique was used according to the attending physician's discretion. The most common concurrent chemotherapy regimen was bolus dosing 5-FU (325 mg/m²/day) with leucovorin $\left(20 \mathrm{mg} / \mathrm{m}^{2} /\right.$ day $)$ for two cycles of five consecutive days at weeks one and five of RT. The second most common concurrent chemotherapy regimen was continuous 5 -FU ( $225 \mathrm{mg} / \mathrm{m}^{2} /$ day) infusion for 6 week continuous cycle.

Surgical resection was scheduled 4-6 weeks after completion of RT. TME was routinely performed in all patients. In cases requiring sphincter preservation surgery, reversal of colostomy or ileostomy was performed approximately 3-6 months after radical surgery. Postoperative chemotherapy was administered according to pathologic staging and physician's discretion.

\section{Outcomes and statistical analysis}

The follow-up duration was calculated as the time from the date of surgery to the date of last review or death. The definition of post-CRT pathologic complete response (pCR) was pT0N0. Down-staging was defined as lowering the $\mathrm{T}$ or $\mathrm{N}$ stage between the pretreatment clinical stage and the post-CRT pathologic stage. Loco-regional recurrence was defined as the presence of tumor in the pelvis or perineum or at the anastomosis site as diagnosed by clinical, radiologic, or pathologic examination. Distant metastasis was defined as evidence of tumor in any other area. Survival rate was calculated as the time from surgery to the date of death, or in the case of survivors, to the date of the last follow-up visit.

Survival rates were estimated by the Kaplan-Meier method and compared using a log-rank test. Parameters evaluated as potential prognostic factors for survival were: tumor response to preoperative CRT, age, gender, tumor distance from anal verge, pretreatment tumor size, tumor circumference extent, RT dose, concurrent chemotherapy regimen, interval between CRT and surgery, and pretreatment clinical stage. All parameters were categorized in two groups according to patient distribution. Parameters with a p-value of less than 0.10 in a univariate analysis were further assessed in a multivariate analysis, using a Cox regression hazard model.

In order to find potential predictive factors for $\mathrm{pCR}$, the following 
parameters were evaluated using a chi-square test: age, gender, tumor distance from anal verge, pretreatment tumor size, tumor circumference extent, RT dose, concurrent chemotherapy regimen, interval between CRT and surgery, and pretreatment clinical stage. Parameters with a p-value of less than 0.10 in a univariate analysis were further assessed in a multivariate analysis, using a multivariate logistic regression model.

For all analyses, a p-value $<0.05$ was considered statistically significant. All analyses were performed using SPSS ver. 15.0 (SPSS Inc., Chicago, IL).

Table 1. Patient characteristics

\begin{tabular}{|c|c|}
\hline Characteristics & No. $(\%)$ \\
\hline Median age (range, yr) & $57(26-86)$ \\
\hline$<60$ & $76(56.3)$ \\
\hline$\geq 60$ & $59(43.7)$ \\
\hline \multicolumn{2}{|l|}{ Gender } \\
\hline Male & $103(76.3)$ \\
\hline Female & $32(23.7)$ \\
\hline Median distance from anal verge (range, $\mathrm{cm}$ ) & $5(1-13)$ \\
\hline$\leq 5$ & $81(60.0)$ \\
\hline$>5$ & $54(40.0)$ \\
\hline Median tumor size (range, $\mathrm{cm}$ ) & $5(1.5-11)$ \\
\hline$<5$ & $66(48.9)$ \\
\hline$\geq 5$ & $69(51.1)$ \\
\hline \multicolumn{2}{|l|}{ Histologic grade } \\
\hline Low & $16(11.8)$ \\
\hline Intermediate & $70(51.9)$ \\
\hline High & $8(5.9)$ \\
\hline Not specified & $41(30.4)$ \\
\hline Median circumference extent (range, $\%$ ) & $80(30-100)$ \\
\hline$\leq 70$ & $60(44.4)$ \\
\hline$>70$ & $75(55.6)$ \\
\hline \multicolumn{2}{|l|}{ Pretreatment clinical stage } \\
\hline $\mathrm{cT} 2$ & $7(5.3)$ \\
\hline cT3 & $98(72.6)$ \\
\hline cT4 & $30(22.1)$ \\
\hline $\mathrm{cN} 0$ & $21(15.5)$ \\
\hline $\mathrm{cN} 1$ & $78(57.8)$ \\
\hline $\mathrm{cN} 2$ & $36(26.7)$ \\
\hline$\leq \mathrm{IIIA}$ & $27(20.0)$ \\
\hline$\geq \mathrm{IIIB}$ & $108(80.0)$ \\
\hline Median RT dose (range, Gy) & $50.4(36-59.4)$ \\
\hline$\leq 50.4$ & $82(60.7)$ \\
\hline$>50.4$ & $53(39.3)$ \\
\hline \multicolumn{2}{|l|}{ Concurrent chemotherapy } \\
\hline 5-fluorouracil & $41(30.4)$ \\
\hline 5-fluorouracil and leucovorin & $94(69.6)$ \\
\hline $\begin{array}{l}\text { Median interval between chemoradiotherapy } \\
\text { and surgery (range, wk) }\end{array}$ & $4.4(0.5-26)$ \\
\hline$\leq 4$ & $56(41.5)$ \\
\hline$>4$ & $79(58.5)$ \\
\hline \multicolumn{2}{|l|}{ Adjuvant chemotherapy } \\
\hline Yes & $67(49.6)$ \\
\hline No & $68(50.4)$ \\
\hline
\end{tabular}

\section{Results}

\section{Patient characteristics}

The study group consisted of 103 males and 32 females. The median patient age was 57 years (range, 26 to 86 years), and the tumor sizes ranged from $1.5 \mathrm{~cm}$ to $11 \mathrm{~cm}$ (median, $5 \mathrm{~cm}$ ). The median distance from the anal verge to the caudal tumor edge was $5 \mathrm{~cm}$ (range, 1 to $13 \mathrm{~cm}$ ), and the most common pretreatment clinical stage was IIIB $(73.5 \%)$. The median RT dose was $50.4 \mathrm{~Gy}$ (range, 36 to $59.4 \mathrm{~Gy}$ ), and the median interval between CRT and surgery was 4.4 weeks (range, 0.5 to 26 weeks). In 2 patients, the resection margin was positive, and 67 patients (49.6\%) received adjuvant chemotherapy. Patient characteristics are summarized in Table 1.

\section{Pathologic findings}

The pT status was $\mathrm{pT} 0$ in 23 patients $(17.0 \%)$, pT1 in 4 patients $(3.0 \%)$, pT2 in 28 patients $(20.7 \%)$, pT3 in 64 patients $(47.4 \%)$, and pT4 in 16 patients $(11.9 \%)$. The $\mathrm{pN}$ status was $\mathrm{pN} 0$ in 102 patients $(75.6 \%), \mathrm{pN} 1$ in 19 patients (14.1\%), and $\mathrm{pN} 2$ in 14 patients (10.3\%). A total of 22 patients (16.3\%) achieved a pCR (pT0N0). In one patient, although the primary tumor had completely regressed, a residual metastatic tumor presented in the perirectal lymph nodes (pT0N+). After CRT, the percentage of T down-staging was $44.4 \%(60 / 135)$ and $\mathrm{N}$ down-staging was $68.2 \%$ (92/135). The percentage of down-staging (T or N) after CRT was $82.2 \%$ (111/135).The pathologic findings are summarized in Table 2.

\section{Treatment outcomes}

The median follow-up time was 50.0 months (range, 4.5 to 157.8 months) for whole patients and 53.3 months (range, 6 to 157.8 months) for surviving patients. Of the whole patients, sphincter preservation surgery was accomplished in 91 patients $(67.4 \%)$, and of the 81 patients

Table 2. Pathologic findings after preoperative chemoradiotherapy

\section{Pathologic findings}

No. $(\%)$

Pathologic stage

pT0

$23 / 135(17.0)$

pT1

$4 / 135(3.0)$

$\mathrm{pT} 2$

$28 / 135(20.7)$

pT3

$64 / 135(47.4)$

pT4

$16 / 135(11.9)$

pNO

$102 / 135(75.6)$

$\mathrm{pN} 1$

$19 / 135$ (14.1)

$\mathrm{pN} 2$

$14 / 135(10.3)$

Pathologic complete response

22/135 (16.3)

T down-staging

60/135 (44.4)

$\mathrm{N}$ down-staging

92/135 (68.2)

Down-staging ( $\mathrm{T}$ or $\mathrm{N}$ )

$111 / 135(82.2)$




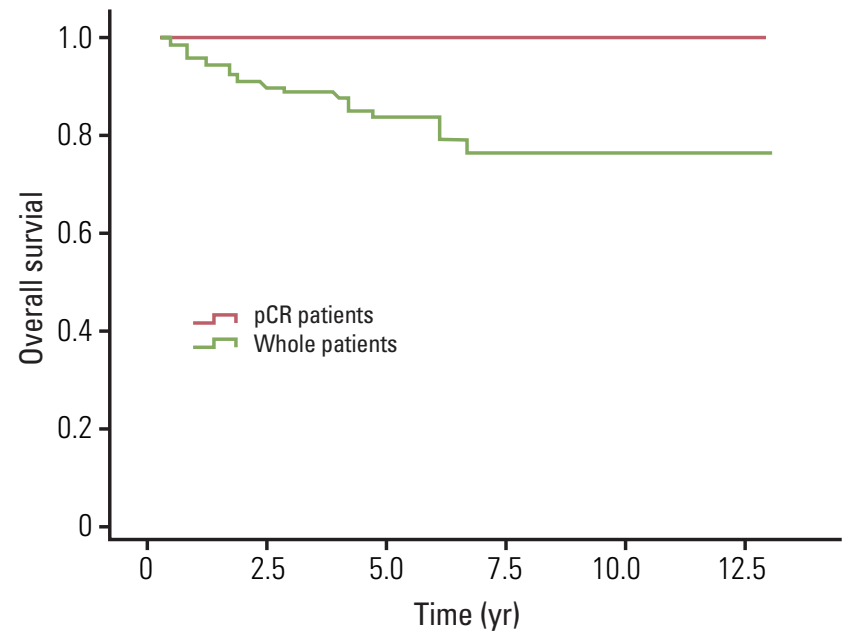

Fig. 1. Overall survival for whole patients and pathologic complete response (pCR) patients. The estimated 5-and 8-year overall survival rates for whole patients were $82.7 \%$ and $75.7 \%$, and for pCR patients were $100 \%$ and $100 \%$, respectively.

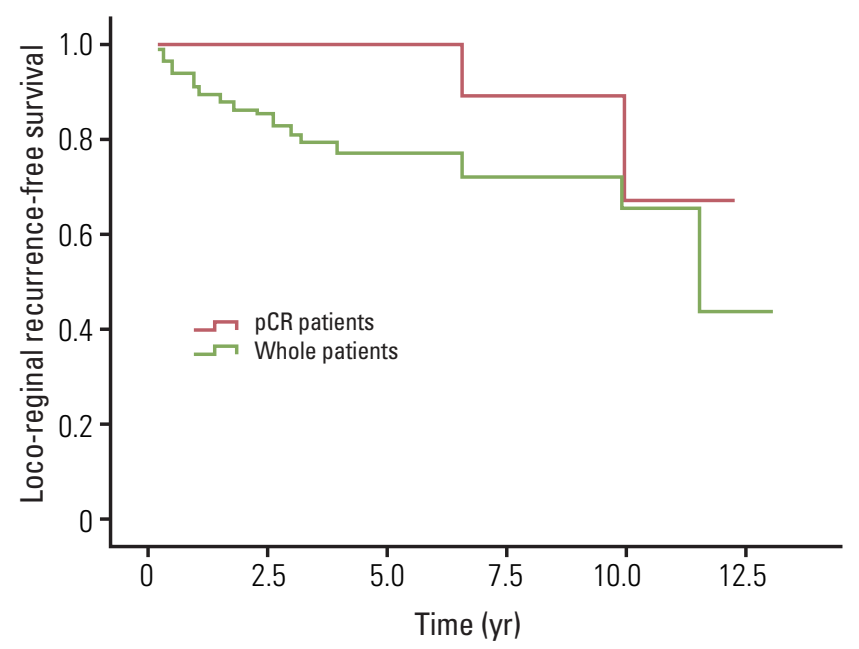

Fig. 2. Loco-regional recurrence-free survival for whole patients and pathologic complete response (pCR) patients. The estimated 5- and 8-year loco-regional recurrence-free survival rates for whole patients were $76.8 \%$ and $71.9 \%$, and for pCR patients were $100 \%$ and $88.9 \%$, respectively.

whose distal tumor edge was $5 \mathrm{~cm}$ or less, sphincter preservation surgery was accomplished in 44 patients (54.3\%). Of those patients who received sphincter preservation surgery, 4 patients received a diverting stoma again due to surgical complications during the follow-up period. Permanent fistula formation developed in two patients, and anastomosis site stenosis occurred in one patient. One other patient received a diverting stoma because of poor healing of recurrent inflammation at the anastomosis site.

The median overall survival was 49.0 months for whole patients and 70.7 months for patients who had a pCR. None of the pCR patients died within the follow-up period. The estimated 5- and 8-year overall survival rates for whole patients were $82.7 \%$ and $75.7 \%$, and for $\mathrm{PCR}$ patients, the
5- and 8-year rates were both $100 \%$ (Fig. 1). The median loco-regional recurrence-free survival was 41.8 months for whole patients and 70.5 months for pCR patients. Loco-regional recurrence developed in 30 $(22.1 \%)$ of the whole patients and $2(9.1 \%)$ of the pCR patients. Of the 30 patients who developed local recurrence, 10 had local recurrence only, and 20 had both local and distant recurrence. Locations of loco-regional recurrence included the anastomosis site $(n=18)$, pelvic wall $(n=6)$, pelvic lymph nodes $(n=3)$, and other areas $(n=3)$. In 2 patients, local recurrences developed 10 years after TME. One patient developed local recurrence in the anastomosis site at 120 months, and the other patient developed in the left pelvic wall at 139.5 months after TME. The estimated 5- and 8year loco-regional recurrence-free survival rates were $76.8 \%$ and $71.9 \%$ for whole patients, and $100 \%$ and $88.9 \%$ for pCR patients (Fig. 2). The median distant metastasis-free survival was 41.0 months for whole patients and 70.5 months for pCR patients. Distant metastasis developed in $39(28.7 \%)$ and 1 (4.5\%) of the whole patients and pCR patients, respectively. Of 39 patients who developed distant metastasis, 19 had a distant metastasis only, and 20 had both local and distant recurrence. Sites of distant metastasis included the liver $(n=20)$, lung $(n=12)$, retroperitoneum $(n=2)$, spine $(n=2)$, and other areas $(n=3)$. The estimated 5- and 8-year distant metastasis-free survival rates were $67.9 \%$ and $63.3 \%$ for whole patients, and $95.5 \%$ and $95.5 \%$ for pCR patients (Fig. 3).

\section{Prognostic factors}

Prognostic factors were analyzed for effect on overall survival, locoregional recurrence-free survival, and distant metastasis-free survival. In the univariate analysis, pCR, T down-staging, down-staging ( $T$ or N), and pretreatment clinical stage were significantly associated with overall sur-

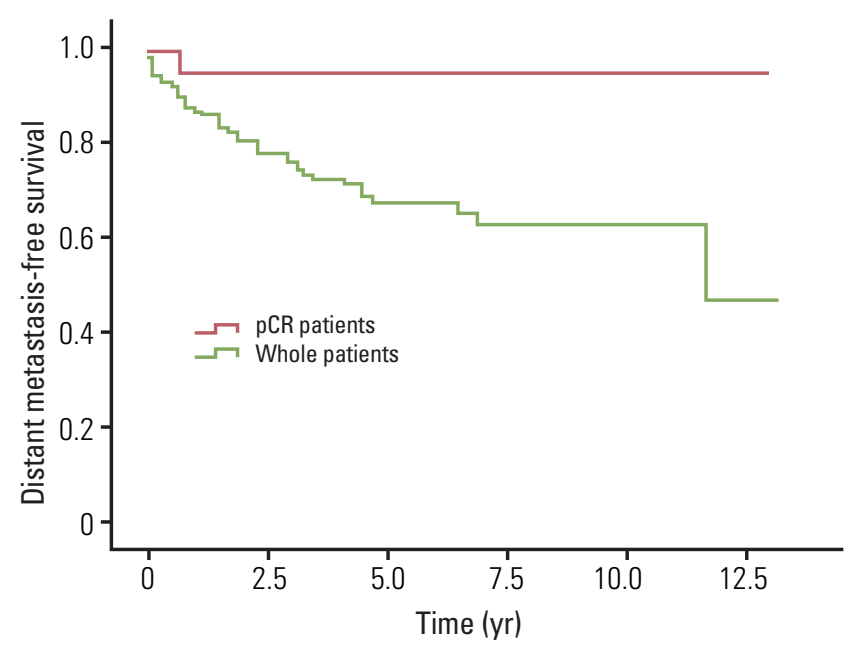

Fig. 3. Distant metastasis-free survival for whole patients and pathologic complete response (pCR) patients. The estimated 5-and 8-year distant metastasis-free survival rates for whole patients were $67.9 \%$ and $63.3 \%$, and for pCR patients were $95.5 \%$ and $95.5 \%$, respectively. 
Table 3. Prognostic factor analysis for overall survival

\begin{tabular}{|c|c|c|c|}
\hline \multirow{2}{*}{ Variables } & \multirow{2}{*}{$\begin{array}{c}\text { 5-year overall } \\
\text { survival rate (\%) }\end{array}$} & \multicolumn{2}{|c|}{ p-value } \\
\hline & & Univariate analysis & Multivariate analysis \\
\hline \multicolumn{4}{|l|}{ Tumor response to preoperative CCRT } \\
\hline pCR vs. pathologic residual disease & 100 vs. 79.1 & 0.021 & 0.048 \\
\hline T down-staging vs. no $\mathrm{T}$ down-staging & 95.3 vs. 71.7 & 0.003 & 0.06 \\
\hline $\mathrm{N}$ down-staging vs. no $\mathrm{N}$ down-staging & 83.5 vs. 73.6 & 0.237 & \\
\hline Down-staging ( $\mathrm{T}$ or $\mathrm{N}$ ) vs. no down-staging & 84.6 vs. 62.9 & 0.024 & 0.15 \\
\hline \multicolumn{4}{|l|}{ Age (yr) } \\
\hline$<60$ vs. $\geq 60$ & 77.1 vs. 86.0 & 0.475 & \\
\hline \multicolumn{4}{|l|}{ Gender } \\
\hline Male vs. Female & 79.9 vs. 90.0 & 0.158 & \\
\hline \multicolumn{4}{|l|}{ Distance from anal verge $(\mathrm{cm})$} \\
\hline$\leq 5$ vs. $>5$ & 81.7 vs. 83.7 & 0.517 & \\
\hline \multicolumn{4}{|l|}{ Pretreatment tumor size $(\mathrm{cm})$} \\
\hline$<5$ vs. $\geq 5$ & 85.7 vs. 80.1 & 0.643 & \\
\hline \multicolumn{4}{|l|}{ Tumor circumference extent $(\%)$} \\
\hline$\leq 70$ vs. $>70$ & 89.8 vs. 76.4 & 0.234 & \\
\hline \multicolumn{4}{|l|}{ RT dose (Gy) } \\
\hline$\leq 50.4$ vs. $>50.4$ & 82.5 vs. 82.6 & 0.856 & \\
\hline \multicolumn{4}{|l|}{ Concurrent chemotherapy regimen } \\
\hline 5-FU alone vs. 5-FU with additional agents & 80.1 vs. 84.1 & 0.581 & \\
\hline 5-FU CI vs. 5-FU BI & 80.1 vs. 84.1 & 0.581 & \\
\hline \multicolumn{4}{|l|}{ Interval between CCRT and surgery (wk) } \\
\hline$\leq 4$ vs. $>4$ & 82.6 vs. 83.3 & 0.918 & \\
\hline \multicolumn{4}{|l|}{ Pretreatment clinical stage } \\
\hline$\leq \mathrm{IIIA}$ vs. $\geq \mathrm{IIIB}$ & 95.2 vs. 77.0 & 0.05 & 0.178 \\
\hline
\end{tabular}

CCRT, concurrent chemoradiotherapy; pCR, pathologic complete response; RT, radiotherapy; 5-FU, 5-fluorouracil; CI, continuous infusion; BI, bolus infusion.

vival. In the multivariate analysis, $\mathrm{pCR}$ associations remained significant (Table 3). In the univariate analysis for loco-regional recurrence-free survival, pCR, T down-staging, and down-staging ( $\mathrm{T}$ or $\mathrm{N}$ ) were significant prognostic factors. In the multivariate analysis, $\mathrm{T}$ down-staging and downstaging (T or N) remained significant factors (Table 4). Many parameters were associated with distant metastasis-free survival. In the univariate analysis, pCR, T down-staging, N down-staging, down-staging (T or N), tumor distance from the anal verge, pretreatment tumor size, and RT dose were significantly associated with distant metastasis-free survival. In the multivariate analysis, pCR, down-staging ( $\mathrm{T}$ or $\mathrm{N})$, pretreatment tumor size, and RT dose remained statistically significant, and pretreatment clinical stage was also found to be significant (Table 5).

Predictive factors for pCR were also analyzed. In the univariate analysis, only pretreatment clinical stage was a significant predictive factor for the pCR rate. Early clinical stage (stage $\leq$ IIIA) was significantly associated with a higher $\mathrm{pCR}$ rate. This factor remained significant in the multivariate analysis (Table 6).

\section{D is c us s i on}

Several reports have found that preoperative CRT or RT alone for patients with locally advanced rectal cancer can decrease local recurrence of rectal cancer $[2,5,10]$. However, results regarding the influence of preoperative CRT or RT on overall survival have conflicted. The Medical Research Council Rectal Cancer Working Party [11] carried out a prospective randomized trial of preoperative RT followed by TME vs. TME alone. They reported an estimated $21 \%$ reduction in the risk of death for patients with preoperative RT followed by TME. However, the overall survival rate was not significantly different between the two groups $(p=0.10)$. Therefore, in that study, preoperative RT did not influence on overall survival. However, Kao et al. [5] reported a 5-year overall survival rate of $88.4 \%$ in a group treated by preoperative CRT followed by TME, and $65.7 \%$ in a group treated by TME alone. In that study, patients with preoperative CRT had a significantly higher overall survival rate. In addition to these studies, several studies reported inconsistent results regarding the influence of preoperative CRT or RT alone on overall survival $[12,13]$. In addition, reported rates of overall survival after preoperative CRT varied widely. In our study, 5- and 8-year overall 
Table 4. Prognostic factor analysis for loco-regional recurrence-free survival

\begin{tabular}{|c|c|c|c|}
\hline \multirow[b]{2}{*}{ Variables } & \multirow{2}{*}{$\begin{array}{l}\text { 5-year loco-regional } \\
\text { recurrence-free } \\
\text { survival rate }(\%)\end{array}$} & \multicolumn{2}{|c|}{ p-value } \\
\hline & & $\begin{array}{c}\text { Univariate } \\
\text { analysis }\end{array}$ & $\begin{array}{c}\text { Multivariate } \\
\text { analysis }\end{array}$ \\
\hline \multicolumn{4}{|l|}{ Tumor response to preoperative CCRT } \\
\hline pCR vs. pathologic residual disease & 100 vs. 72.0 & 0.05 & 0.057 \\
\hline T down-staging vs. no $\mathrm{T}$ down-staging & 91.3 vs. 64.4 & 0.005 & 0.007 \\
\hline $\mathrm{N}$ down-staging vs. no $\mathrm{N}$ down-staging & 80.2 vs. 73.3 & 0.097 & 0.091 \\
\hline Down-staging ( $\mathrm{T}$ or $\mathrm{N}$ ) vs. no down-staging & 82.6 vs. 50.2 & 0.002 & 0.004 \\
\hline \multicolumn{4}{|l|}{ Age (yr) } \\
\hline$<60$ vs. $\geq 60$ & 76.0 vs. 78.2 & 0.89 & \\
\hline \multicolumn{4}{|l|}{ Gender } \\
\hline Male vs. Female & 73.4 vs. 86.2 & 0.115 & \\
\hline \multicolumn{4}{|l|}{ Distance from anal verge $(\mathrm{cm})$} \\
\hline$\leq 5$ vs. $>5$ & 72.2 vs. 83.9 & 0.118 & \\
\hline \multicolumn{4}{|l|}{ Pretreatment tumor size $(\mathrm{cm})$} \\
\hline$<5$ vs. $\geq 5$ & 83.4 vs. 70.1 & 0.186 & \\
\hline \multicolumn{4}{|l|}{ Tumor circumference extent $(\%)$} \\
\hline$\leq 70$ vs. $>70$ & 81.5 vs. 74.0 & 0.129 & \\
\hline \multicolumn{4}{|l|}{ RT dose (Gy) } \\
\hline$\leq 50.4$ vs. $>50.4$ & 78.7 vs. 72.9 & 0.356 & \\
\hline \multicolumn{4}{|l|}{ Concurrent chemotherapy regimen } \\
\hline 5-FU alone vs. 5-FU with additional agents & 77.7 vs. 76.5 & 0.832 & \\
\hline 5-FU CI vs. 5-FU BI & 77.7 vs. 76.5 & 0.832 & \\
\hline \multicolumn{4}{|l|}{ Interval between CCRT and surgery (wk) } \\
\hline$\leq 4$ vs. $>4$ & 77.5 vs. 76.3 & 0.585 & \\
\hline \multicolumn{4}{|l|}{ Pretreatment clinical stage } \\
\hline$\leq \mathrm{IIIA}$ vs. $\geq \mathrm{IIIB}$ & 85.1 vs. 74.8 & 0.221 & \\
\hline
\end{tabular}

CCRT, concurrent chemoradiotherapy; pCR, pathologic complete response; RT, radiotherapy; 5-FU, 5-fluorouracil; CI, continuous infusion; BI, bolus infusion.

survival rates were $82.7 \%$ and $75.7 \%$, respectively. However, numerous studies [1,8,9,14-17] have reported inconsistent overall survival rates (Table 7). Possible reasons for these inconsistent results include different preoperative CRT regimens, different indications and regimens for postoperative chemotherapy, and inconsistent interpretation of radiologic imaging studies by different radiologists.

The reported rate of sphincter preservation surgery after preoperative CRT has also varied widely, ranging from $28 \%$ to $89 \%[1,2,15]$. However, regardless of the sphincter preservation surgery rate, several studies have reported that preoperative CRT significantly increases the rate of sphincter preservation surgery as compared to patients not receiving preoperative CRT or RT $[2,18]$. The reported rate of sphincter preservation surgery in our study was relatively high, with $67.4 \%$ of the whole patients and $54.3 \%$ of the patients whose distal tumor edge was $5 \mathrm{~cm}$ or less. However, Kao et al. [5] have argued that in order to confirm the influence of preoperative CRT on sphincter preservation, one should compare the rates of preserved anorectal function after long-term follow-up rather than the rates of sphincter preservation surgery. In that study, patients with preoperative CRT followed by TME had a significantly higher rate of sphincter preservation surgery as compared to patients receiving TME only (75.4\% vS. $50.7 \%$, $\mathrm{p}=0.005$ ). However, the rate of surgical complications, such as anastomosis stenosis and fistula formation was relatively higher in the preoperative CRT group after long-term follow-up. As some patients received a diverting stoma again due to surgical complications, in the final results, $62.3 \%$ of the patients with preoperative CRT followed by TME and $47.8 \%$ of patients with TME alone maintained preserved anorectal function $(\mathrm{p}=0.125)$. Therefore, although the rate of sphincter preservation surgery was significantly higher in patients with preoperative CRT followed by TME, there was no statistical difference between the two groups with respect to preservation of anorectal function at the time of the last follow-up. In our study, 4 patients received a diverting stoma again due to surgical complications including poor healing of recurrent inflammation at the anastomosis site (1 patient), permanent fistula formation (2 patients), and anostomosis stenosis (1 patient). Because our study was not a randomized comparative study, we could not verify the influence of preoperative CRT on sphincter preservation.

The definition of $\mathrm{pCR}$ after preoperative CRT varies between $95 \%$ or more primary tumor response, pT0 only, or pT0N0 [8,19,20]. However, tumor regression after preoperative CRT may be observed not only in the primary tumor, but also in the perirectal metastatic lymph nodes, and the 
Table 5. Prognostic factor analysis for distant metastasis-free survival

\begin{tabular}{|c|c|c|c|}
\hline \multirow[b]{2}{*}{ Variables } & \multirow{2}{*}{$\begin{array}{c}\text { 5-year distant } \\
\text { metastasis-free } \\
\text { survival rate (\%) }\end{array}$} & \multicolumn{2}{|c|}{ p-value } \\
\hline & & $\begin{array}{c}\text { Univariate } \\
\text { analysis }\end{array}$ & $\begin{array}{c}\text { Multivariate } \\
\text { analysis }\end{array}$ \\
\hline \multicolumn{4}{|l|}{ Tumor response to preoperative CCRT } \\
\hline pCR vs. pathologic residual disease & 95.5 vs. 62.1 & 0.005 & 0.019 \\
\hline T down-staging vs. no $\mathrm{T}$ down-staging & 84.2 vs. 48.6 & 0.001 & 0.065 \\
\hline $\mathrm{N}$ down-staging vs. no $\mathrm{N}$ down-staging & 74.6 vs. 54.0 & 0.002 & 0.263 \\
\hline Down-staging ( $\mathrm{T}$ or $\mathrm{N}$ ) vs. no down-staging & 75.8 vs. 35.3 & $<0.001$ & $<0.001$ \\
\hline \multicolumn{4}{|l|}{ Age (yr) } \\
\hline$<60$ vs. $\geq 60$ & 60.5 vs. 78.2 & 0.111 & \\
\hline \multicolumn{4}{|l|}{ Gender } \\
\hline Male vs. Female & 66.5 vs. 70.6 & 0.465 & \\
\hline \multicolumn{4}{|l|}{ Distance from anal verge $(\mathrm{cm})$} \\
\hline$\leq 5$ vs. $>5$ & 61.3 vs. 77.7 & 0.05 & 0.099 \\
\hline \multicolumn{4}{|l|}{ Pretreatment tumor size (cm) } \\
\hline$<5$ vs. $\geq 5$ & 79.2 vs. 57.6 & 0.011 & 0.023 \\
\hline \multicolumn{4}{|l|}{ Tumor circumference extent (\%) } \\
\hline$\leq 70$ vs. $>70$ & 73.4 vs. 63.8 & 0.114 & \\
\hline \multicolumn{4}{|l|}{ RT dose (Gy) } \\
\hline$\leq 50.4$ vs. $>50.4$ & 75.0 vs. 52.2 & 0.05 & 0.042 \\
\hline \multicolumn{4}{|l|}{ Concurrent chemotherapy regimen } \\
\hline 5-FU alone vs. 5-FU with additional agents & 75.1 vs. 64.5 & 0.184 & \\
\hline 5-FU CI vs. 5-FU BI & 75.1 vs. 64.5 & 0.184 & \\
\hline \multicolumn{4}{|l|}{ Interval between CCRT and surgery (wk) } \\
\hline$\leq 4$ vs. $>4$ & 76.3 vs. 60.4 & 0.359 & \\
\hline \multicolumn{4}{|l|}{ Pretreatment clinical stage } \\
\hline$\leq$ IIIA vs. $\geq$ IIIB & 86.7 vs. 63.4 & 0.069 & 0.038 \\
\hline
\end{tabular}

CCRT, concurrent chemoradiotherapy; pCR, pathologic complete response; RT, radiotherapy; 5-FU, 5-fluorouracil; CI, continuous infusion; BI, bolus infusion.

observation of complete primary tumor regression (pT0) does not guarantee complete nodal sterilization. Actually, the rate of nodal disease $(\mathrm{pN}+)$ in patients with complete primary tumor regression (pT0) has been reported be as high as $17 \%$ [21]. In addition, several studies have reported that $\mathrm{pN}$ status is a significant prognostic factor for long-term outcome in locally advanced rectal cancer patients. Coco et al. [9] reported a 10-year overall survival rate of $66 \%$ in $\mathrm{pN} 0$ patients and $42 \%$ in $\mathrm{pN}+$ patients ( $p=0.003$ ), and Shivnani et al. [1] reported 5-year disease-free survival rates of $79 \%$ in $\mathrm{pN} 0-1$ patients and $25 \%$ in $\mathrm{pN} 2$ patients $(\mathrm{p}=0.002)$. Yeo et al. [3] investigated the long-term outcomes of rectal cancer patients with pT0 following preoperative CRT and determined the prognostic significance of $\mathrm{pN}$ status. In that study, the 5-year disease-free survival and overall survival rates in pT0N0 were $88.5 \%$ and $94.8 \%$, and in $\mathrm{pT} 0 \mathrm{~N}+$ patients were $45.2 \%$ and $72.8 \%$, respectively $(\mathrm{p}<0.001)$. These findings indicated that a pCR group defined only by primary tumor response (pT0) may contain $\mathrm{pT} 0 \mathrm{~N}+$ patients, and these $\mathrm{pT} 0 \mathrm{~N}+$ patients have significantly different long-term outcomes. Therefore, we believe the definition of $\mathrm{pCR}$ after preoperative CRT should be pT0N0. In our study, similar to Yeo et al.'s study [3], the 5- and 8-year overall survival rates, loco-regional recurrence-free survival rates, and distant metastasis-free survival rates for pCR patients were $100 \%$ and $100 \%, 100 \%$ and $88.9 \%, 95.5 \%$ and $95.5 \%$, respectively.

Although many physicians follow an intensive surveillance program for at least 5 years in cases of rectal cancer, recommendations for the postoperative surveillance period after preoperative CRT followed by TME remain unclear. Coco et al. [9] reported that $28 \%$ of local recurrence and $9.5 \%$ of distant metastasis developed 5 years after TME in rectal cancer patients receiving preoperative CRT, and Guillem et al. [8] reported that all local recurrence developed 5 years after TME. In our study, 16.7\% (5/30) of local recurrence and $12.8 \%$ (5/39) of distant metastasis developed 5 years after curative TME. These findings suggest that preoperative CRT is capable of delaying recurrence in rectal cancer patients, and a surveillance period longer than 5 years is warranted for a complete assessment of the outcome of rectal cancer treated by preoperative CRT followed by curative rectal resection. In order to confirm the effectiveness of specific postoperative surveillance programs, multicenter trials with large sample sizes and long-term follow-up periods will be required to better define the patterns of loco-regional and distant recurrence for rectal cancer patients treated with preoperative CRT followed by TME.

In the management of patients with locally advanced rectal cancer, 
Table 6. Predictive factor analysis for $\mathrm{pCR}$

\begin{tabular}{|c|c|c|c|}
\hline \multirow{2}{*}{ Variables } & \multirow{2}{*}{ pCR rate (\%) } & \multicolumn{2}{|c|}{ p-value } \\
\hline & & Univariate analysis & Multivariate analysis \\
\hline \multicolumn{4}{|l|}{ Age (yr) } \\
\hline$<60$ vs. $\geq 60$ & 44.1 vs. 55.9 & 0.741 & \\
\hline \multicolumn{4}{|l|}{ Gender } \\
\hline Male vs. Female & 24.3 vs. 75.7 & 0.367 & \\
\hline \multicolumn{4}{|l|}{ Distance from anal verge $(\mathrm{cm})$} \\
\hline$\leq 5$ vs. $>5$ & 40.4 vs. 59.6 & 0.670 & \\
\hline \multicolumn{4}{|l|}{ Pretreatment tumor size $(\mathrm{cm})$} \\
\hline$<5$ vs. $\geq 5$ & 50.7 vs. 49.3 & 0.141 & \\
\hline \multicolumn{4}{|l|}{ Tumor circumference extent (\%) } \\
\hline$\leq 70$ vs. $>70$ & 55.9 vs. 44.1 & 0.122 & \\
\hline \multicolumn{4}{|l|}{ RT dose (Gy) } \\
\hline$\leq 50.4$ vs. $>50.4$ & 39.7 vs. 60.3 & 0.726 & \\
\hline \multicolumn{4}{|l|}{ Concurrent chemotherapy regimen } \\
\hline 5-FU alone vs. 5-FU with addition agents & 30.1 vs. 69.9 & 0.182 & \\
\hline 5-FU CI vs. 5-FU BI & 30.1 vs. 69.9 & 0.182 & \\
\hline \multicolumn{4}{|l|}{ Interval between CCRT and surgery (wk) } \\
\hline$\leq 4$ vs. $>4$ & 41.9 vs. 58.1 & 0.565 & \\
\hline \multicolumn{4}{|l|}{ Pretreatment clinical stage } \\
\hline$\leq$ IIIA vs. $\geq$ IIIB & 80.1 vs. 19.9 & 0.007 & 0.021 \\
\hline
\end{tabular}

pCR, pathologic complete response; RT, radiotherapy; 5-FU, 5-fluorouracil; CI, continuous infusion; BI, bolus infusion.

Table 7. Reported overall survival rate in various studies

\begin{tabular}{lcc} 
Authors & Sample size (n) & $\begin{array}{c}\text { Reported overall } \\
\text { survival rate (\%) }\end{array}$ \\
\hline Rullier et al. [14] & 43 & 3 -yr, 85.0 \\
Shivnani et al. [1] & 100 & $5-\mathrm{yr}, 81.0$ \\
Grann et al. [15] & 72 & $5-\mathrm{yr}, 88.0$ \\
Osti et al. [16] & 140 & 5 -yr, 71.3 \\
Coco et al. [9] & 83 & 5 -yr, 75.5 \\
& & $7-\mathrm{yr}, 67.8$ \\
Guillem et al. [8] & 297 & $10-\mathrm{yr}, 60.4$ \\
& & $5-\mathrm{yr}, 76.0$ \\
& & $7-\mathrm{yr}, 63.0$ \\
Gérard et al. [17] & 375 & $0-\mathrm{yr}, 58.0$ \\
Kong et al. (this study) & 136 & $5-\mathrm{yr}, 67.4$ \\
& & $5-\mathrm{yr}, 82.7$ \\
\hline
\end{tabular}

perhaps no topic is as controversial as the prognostic factors for survival after preoperative CRT. Numerous studies have reported prognostic factors significantly associated with survival, but most were retrospective or small phase II studies, and their results were inconsistent. There have been no large, prospective phase III clinical trials which evaluate prognostic factors until now. However, from among the various prognostic factors, pCR after preoperative CRT has been accepted in several studies as significant prognostic factor for survival $[3,8]$. Likewise, in our study,
pCR was a significant prognostic factor for overall survival, loco-regional recurrence-free survival, and distant metastasis-free survival in univariate analysis. After multivariate analysis, pCR remained a significant factor for overall survival and distant metastasis-free survival. Several studies reported highly favorable results for rectal cancer patients showing pCR after preoperative CRT $[8,19,20]$. Furthermore, Habr-Gama and Perez $[22,23]$ reported that post-CRT clinical complete responders who were managed by observation alone, with no surgical resection, had oncologic outcomes similar to those managed by preoperative CRT followed by radical surgery. So, Habr-Gama and Perez [22,23] proposed that clinical complete responders after CRT should receive no surgery but be subjected to close observation instead. With these findings in mind, identification of predictive factors for $\mathrm{pCR}$ is important for predicting treatment outcomes and developing risk-adapted treatment. A low pretreatment carcinoembryonic antigen level, use of 5-FU in a continuous venous infusion fashion, delivery of RT dose higher than $45 \mathrm{~Gy}$, use of a second drug in addition to 5-FU, and longer intervals between CRT and surgery were reported to be significant predictive factors for pCR [24,25]. In our study, pretreatment clinical stage was a significant predictive factor for pCR. In patients who received RT doses higher than 50.4 Gy and who received concurrent chemotherapy using 5-FU plus additional agents, the pCR rate after preoperative CRT was higher, but not statistically significant.

There were some limitations in this study. First, this study was retrospective, and therefore, may have inherent bias. For example, postoperative chemotherapy was provided according to the attending physician's 
discretion rather than a predetermined protocol. Thus, the number of patients who received postoperative chemotherapy was small. Second, the sample size was relatively small. Third, the duration of the follow-up period was not sufficiently long in some cases, and consequently, this study may underestimate the cancer recurrence rate. Despite these limitations, we believe that our study contributes to resolving several controversial issues in rectal cancer management.

\section{Conclusion}

In our study, preoperative CRT for locally advanced rectal cancer resulted in a high rate of overall survival, sphincter preservation, downstaging, and pCR. We defined pCR as pTON0, and the patients achieving pCR after preoperative CRT had very favorable outcomes (5- and 8-year overall survival rates were both $100 \%$ ). pCR was found to be a significant prognostic factor for both overall and distant metastasis-free survival. The significant predictive factor for $\mathrm{pCR}$ was pretreatment clinical stage.

\section{Conflicts of Interest}

Conflict of interest relevant to this article was not reported.

\section{References}

1. Shivnani AT, Small W Jr, Stryker SJ, Kiel KD, Lim S, Halverson AL, et al. Preoperative chemoradiation for rectal cancer: results of multimodality management and analysis of prognostic factors. Am J Surg. 2007:193:389-93.

2. Sauer R, Becker H, Hohenberger W, Rödel C, Wittekind C, Fietkau R, et al. Preoperative versus postoperative chemoradiotherapy for rectal cancer. N Engl J Med. 2004;351:1731-40.

3. Yeo SG, Kim DY, Kim TH, Chang HJ, Oh JH, Park W, et al. Pathologic complete response of primary tumor following preoperative chemoradiotherapy for locally advanced rectal cancer: long-term outcomes andprognostic significance of pathologic nodal status (KROG 09-01). Ann Surg. 2010;252:998-1004.

4. Wheeler JM, Dodds E, Warren BF, Cunningham C, George BD, Jones AC, et al. Preoperative chemoradiotherapy and total mesorectal excision surgery for locally advanced rectal cancer: correlation with rectal cancer regression grade. Dis Colon Rectum. 2004;47:2025-31.

5. Kao PS, Chang SC, Wang LW, Lee RC, Liang WY, Lin TC, et al. The impact of preoperative chemoradiotherapy on advanced low rectal cancer. J Surg Oncol. 2010;102:771-7.

6. Kapiteijn E, Marijnen CA, Nagtegaal ID, Putter H, Steup WH, Wiggers T, et al. Preoperative radiotherapy combined with total mesorectal excision for resectable rectal cancer. N Engl J Med. 2001;345:638-46.

7. Improved survival with preoperative radiotherapy in resectable rectal cancer. Swedish Rectal Cancer Trial. N Engl J Med. 1997;336:980-7.

8. Guillem JG, Chessin DB, Cohen AM, Shia J, Mazumdar M, Enker W, et al. Long-term oncologic outcome following preoperative combined modality therapy and total mesorectal excision of locally advanced rectal cancer. Ann Surg. 2005;241:829-36.

9. Coco C, Valentini V, Manno A, Mattana C, Verbo A, Cellini N, et al. Long-term results after neoadjuvant radiochemotherapy for locally advanced resectable extra peritoneal rectal cancer. Dis Colon Rectum. 2006:49:311-8.

10. Bosset JF, Collette L, Calais G, Mineur L, Maingon P, Radosevic-Jelic L, et al. Chemotherapy with preoperative radiotherapy in rectal cancer. N Engl J Med. 2006;355:1114-23.

11. Randomised trial of surgery alone versus radiotherapy followed by surgery for potentially operable locally advanced rectal cancer. Medical Research Council Rectal Cancer Working Party. Lancet. 1996;348:1605-10.

12. Cammà C, Giunta M, Fiorica F, Pagliaro L, Craxi A, Cottone M. Preoperative radiotherapy for resectable rectal cancer: a meta-analysis. JAMA. 2000;284:1008-15.

13. Roh MS, Colangelo LH, O'Connell MJ, Yothers G, Deutsch M, Allegra CJ, et al. Preoperative multimodality therapy improves disease-free survival in patients with carcinoma of the rectum: NSABP R-03. J Clin Oncol. 2009;27:5124-30.

14. Rullier E, Goffre B, Bonnel C, Zerbib F, Caudry M, Saric J. Preoperative radiochemotherapy and sphincter-saving resection for T3 carcinomas of the lower third of the rectum. Ann Surg. 2001;234:633-40.

15. Grann A, Feng C, Wong D, Saltz L, Paty PP, Guillem JG, et al. Preoperative combined modality therapy for clinically resectable uT3 rectal adenocarcinoma. Int J Radiat Oncol Biol Phys. 2001:49:987-95.

16. Osti MF, Valeriani M, Masoni L, Tombolini V, Enrici RM. Neoadjuvant chemoradiation for locally advanced carcinoma of the rectum. Tumori. 2004;90:303-9.

17. Gérard JP, Conroy T, Bonnetain F, Bouché 0, Chapet 0, Closon-Dejardin MT, et al. Preoperative radiotherapy with or without concurrent fluorouracil and leucovorin in T3-4 rectal cancers: results of FFCD 9203. J Clin Oncol. 2006;24:4620-5.

18. Howard JH, Gonzalez Q, Arnoletti JP, Russo S, Fiveash JB, Bland KI, et al. Prognostic factors and preoperative radiation therapy associated with sphincter preservation in patients with resectable rectal cancer. Am J Surg. 2008;195:239-43.

19. Ruo L, Tickoo S, Klimstra DS, Minsky BD, Saltz L, Mazumdar M, et al. Long-term prognostic significance of extent of rectal cancer response to preoperative radiation and chemotherapy. Ann Surg. 2002;236:75-81

20. García-Aguilar J, Hernandez de Anda E, Sirivongs P, Lee SH, Madoff RD, Rothenberger DA. A pathologic complete response to preoperative chemoradiation is associated with lower local recurrence and improved survival in rectal cancer patients treated by mesorectal excision. Dis Colon Rectum. 2003:46:298-304.

21. Hughes R, Glynne-Jones R, Grainger J, Richman P, Makris A, Harrison M, et al. Can pathological complete response in the primary tumour following pre-operative pelvicchemoradiotherapy for T3-T4 rectal cancer predict for sterilisation of pelvic lymph nodes, a low risk of local recurrence and the appropriateness of local excision? Int J Colorectal Dis. 2006;21:117.

22. Habr-Gama A. Assessment and management of the complete clinical response of rectal cancer to chemoradiotherapy. Colorectal Dis. 2006;8 Suppl 3:21-4.

23. Habr-Gama A, Perez RO. Non-operative management of rectal cancer after neoadjuvant chemoradiation. Br J Surg. 2009:96:125-7.

24. Sanghera P, Wong DW, McConkey CC, Geh JI, Hartley A. Chemoradiotherapy for rectal cancer: an updated analysis of factors affecting pathological response. Clin Oncol (R Coll Radiol). 2008:20:176-83.

25. Kalady MF, de Campos-Lobato LF, Stocchi L, Geisler DP, Dietz D, Lavery IC, et al. Predictive factors of pathologic complete response after neoadjuvant chemoradiation for rectal cancer. Ann Surg. 2009;250:582-9. 